\title{
Uplink Load Estimates in WCDMA with Different Availability of Measurements
}

\author{
Erik Geijer Lundin, Fredrik Gunnarsson, Fredrik Gustafsson \\ Division of Communication Systems \\ Department of Electrical Engineering \\ Linköpings universitet, SE-581 83 Linköping, Sweden \\ WWW: http://www. control.isy.liu.se \\ E-mail: geijer@isy.liu.se, fred@isy.liu.se \\ fredrik@isy.liu.se
}

9th September 2003

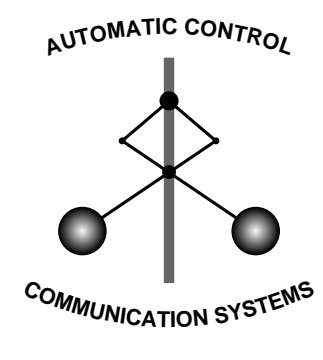

LINKÖPING

Report no.: LiTH-ISY-R-2544

Submitted to VTC'03 Spring

Technical reports from the Control \& Communication group in Linköping are available at http://www. control.isy.liu.se/publications. 


\begin{abstract}
In the uplink of a WCDMA system, a natural choice of resource management control quantity is the uplink noise rise, i.e., total received power over noise power. Unfortunately this quantity is hard to measure. In this paper, we propose and evaluate a number of noise rise estimates which all rely on path gain measurements. These measurements can be made available either periodically or event-driven as described in 3GPP (Release 99). Simulations show that event-driven measurements yield comparable performance to periodic measurements, but with much fewer measurement reports. Despite severely limited path gain knowledge due to that some users report to another $\mathrm{RNC}$, we still manage to estimate the uplink noise rise reasonably well.
\end{abstract}

Keywords: WCDMA, Uplink Load, Handover 


\title{
Uplink Load Estimates in WCDMA with Different Availability of Measurements
}

\author{
Erik Geijer Lundin, Fredrik Gunnarsson and Fredrik Gustafsson* \\ Division of Communication Systems \\ Department of Electrical Engineering, Linköpings universitet \\ SE-581 83 LINKÖPING, SWEDEN \\ \{geijer, fred, fredrik\}@isy.liu.se
}

\begin{abstract}
In the uplink of a WCDMA system, a natural choice of resource management control quantity is the uplink noise rise, i.e., total received power over noise power. Unfortunately this quantity is hard to measure. In this paper, we propose and evaluate a number of noise rise estimates which all rely on path gain measurements. These measurements can be made available either periodically or event-driven as described in 3GPP (Release 99). Simulations show that event-driven measurements yield comparable performance to periodic measurements, but with much fewer measurement reports. Despite severely limited path gain knowledge due to that some users report to another RNC, we still manage to estimate the uplink noise rise reasonably well.
\end{abstract}

\section{INTRODUCTION}

All cellular radio systems are equipped with radio resource management algorithms. In the uplink of a WCDMA system a natural choice of control quantity for these algorithms is the uplink noise rise, i.e., total received uplink power over background noise power. Interference power measures are used for admission control in $[1,2]$. In the literature there are several ways of estimating the affect that admitting a new user has on the uplink noise rise. In for example [3] they estimate the noise rise increase by using measured current uplink noise rise and an estimate of the increase in relative load a new user will provide. A common approach to estimating the uplink interference power is to divide it into background noise, intracell interference (interference originating from the own cell) and inter-cell interference (interference from other cells). In [4] they measure the inter-cell interference power periodically and estimates the intra-cell interference as a sum of contributions from each user within the cell. Another, perhaps more common approach, is to assume that the inter-cell interference power is a fraction of the intra-cell interference power. This technique is used in e.g., [5, 6, 7]. Our approach is to assume that the estimate will be hosted in the Radio Network Controller (RNC) and therefore have knowledge of the situation in several cells. The uplink interference power can than be modeled as background noise plus a sum of each user's contribution, regardless of whether they are within or outside the cell. The estimates rely heavily on somewhat accurate knowledge of the path gain between base stations and users. Therefore, it is interesting to see how sensitive they are to incomplete path gain knowledge. We have studied two different manners in which the path gain measurements are reported by the users; once in every two-second period (which will simulate

* This work is supported by the Swedish Agency for Innovation Systems (VINNOVA), Information Systems for Industrial Control and Supervision (ISIS) and in cooperation with Ericsson Research, which are all acknowledged. periodically requested reports) and event-driven report instants where the user's path gains are reported only in conjunction with handover requests. In [8] they conclude that the system quality is independent of the type of report scheduling used, event-driven or periodical, and that using event-driven reports results in less signaling load. We have also studied the case where some of the users reports only to another RNC.

The outline of the rest of this paper is as follows. Section II contains a derivation of the estimates used herein. In Section III we define the two types of limitations which will be put on the measurement availability. The estimates' sensitivity to these limitations is then investigated through simulations in Section IV. Finally, some conclusions are drawn in Section V.

\section{SYSTEM MODEL}

In this section we will present a number of estimates of the uplink noise rise, $\Lambda$, which in [3] is related to the uplink load by the well known pole equation

$$
\begin{gathered}
L \triangleq 1-\frac{N}{I^{t o t}} \\
\Lambda \triangleq \frac{I^{t o t}}{N}=\frac{1}{1-L}
\end{gathered}
$$

where $I^{t o t}, N$ and $L$ are the total received interference power, the background noise power and the uplink relative load, respectively. The estimates are based on knowledge of the current path gains in the network. We will later evaluate how sensitive the estimates are with respect to incomplete knowledge regarding the path gain matrix.

$I^{\text {tot }}$ can be seen as a sum of background noise and the sum of all users' signals, i.e.,

$$
I_{j}^{t o t}(t)=N_{j}(t)+\sum_{i=1}^{M} p_{i}(t) g_{i, j}(t)
$$

where $g_{i, j}(t)(<1)$ is the path gain between user $i$ and the base station serving cell $j, p_{i}(t)$ is user $i$ 's momentary transmission power and $M$ is the number of users in the entire network. WCDMA utilizes macro diversity since one user can be connected to several cells at a time. If we assume that the signals received in different cells from user $i$ are combined using maximum ratio combining (i.e., utilizing softer handover), the carrier-to-total-interference-ratio ${ }^{1}, \beta_{i}=\frac{C_{i}}{I_{i}^{\text {tot }}}$,

\footnotetext{
${ }^{1}$ Note the close relation with the more common CIR, $\beta=\frac{\gamma}{1+\gamma}$
} 
is the sum of the received signal qualities

$$
\beta_{i}=p_{i} \sum_{k \in K_{i}} \frac{g_{i, k}}{I_{k}^{t o t}} \Leftrightarrow p_{i}=\frac{\beta_{i}}{\sum_{k \in K_{i}} \frac{g_{i, k}}{I_{k}^{t o t}}}
$$

where $K_{i}$ is the set of cells user $i$ is connected to. Even though the above summation is in reality done in another time scale then assumed here, the approximation is good enough for our purposes. Assuming perfect power control $\left(\beta_{i}=\beta_{i}^{t g t}\right)$ and inserting the above estimate of user $i$ 's transmission power yield

$$
\begin{array}{r}
I_{j}^{t o t}(t)=N_{j}(t)+\sum_{i=1}^{M} g_{i, j}(t) p_{i}(t)= \\
N_{j}(t)+\sum_{i=1}^{M} g_{i, j}(t) \frac{\beta_{i}^{t g t}(t)}{\sum_{k \in K_{i}} \frac{g_{i, k}(t)}{I_{k}^{t o t}(t)}}
\end{array}
$$

This defines a set of nonlinear equations in $I^{t o t}(t)$. We use two different ways of solving these equations.

\section{A. Assuming equal background noise power in neighbouring cells}

If the the background noise power in the different cells can be considered equal, i.e., $N_{i}=N_{k}=N$, we may divide equation (4) with that $N$ and substitute $\frac{I_{j}}{N}$ by $\hat{\Lambda}_{j}$

$$
\hat{\Lambda}_{j}(t)=1+\sum_{i=1}^{M} \beta_{i}^{t g t}(t) \frac{g_{i, j}(t)}{\sum_{k \in K_{i}} \frac{g_{i, k}(t)}{\hat{\Lambda}_{k}(t)}}
$$

We may solve this nonlinear system numerically through fixpoint iterations, i.e.,

$$
\begin{aligned}
& \text { Let } \hat{\Lambda}(t, 0)=\hat{\Lambda}(t-1) \\
& \text { Iterate in } n \text { until convergence } \\
& \text { Let } \hat{\Lambda}(t, n)=u\left(g, \beta^{t g t}, \hat{\Lambda}(t, n-1)\right) \\
& \hat{\Lambda}(t)=\hat{\Lambda}(t, n)
\end{aligned}
$$

where $u$ corresponds to equation (5). By only iterating once, we get the following recursive algorithm.

$$
\hat{\Lambda}_{j}^{(N M R C)}(t)=1+\sum_{i=1}^{M} \beta_{i}^{t g t}(t) \frac{g_{i, j}(t)}{\sum_{k \in K_{i}} \frac{g_{i, k}(t)}{\hat{\Lambda}_{k}^{(N M R C)}(t-1)}}
$$

Where the index $(N M R C)$ indicates that maximum ratio combining has been used when combining the information from several receivers and that we have assumed that $N_{k}=$ $N_{j}$. In equation (6) we assumed softer handover is used for all macro diversity connections. This may seem a bit too optimistic. Instead we can either assume soft handover is used for all macro diversity connections (resulting in selection combining of the received signals, soft handover)

$$
\hat{\Lambda}_{j}^{(N S C)}(t)=1+\sum_{i=1}^{M} \beta_{i}^{t g t}(t) \frac{g_{i, j}(t)}{\max _{k \in K_{i}} \frac{g_{i, k}(t)}{\hat{\Lambda}_{k}^{(N S C)}(t-1)}}
$$

or use the actual combination of soft and softer handover

$$
\hat{\Lambda}_{j}^{(N B O T H)}(t)=1+\sum_{i=1}^{M} \beta_{i}^{t g t}(t) \frac{g_{i, j}(t)}{f\left(\frac{g(t)}{\hat{\Lambda}^{(N B O T H)}(t-1)}, K_{i}(t)\right)}
$$

Here, $f$ is a function that considers the information regarding the combination of soft and softer handover defined by $K_{i}(t)$.

\section{B. Assuming equal interference power in neighbouring cells}

Another approach to solving equation (4) is to assume that $I_{k}^{t o t}(t) \approx I_{j}^{t o t}(t)$. The equation then becomes

$$
\begin{gathered}
\hat{I}_{j}^{t o t}(t)=N_{j}(t)+\hat{I}_{j}^{t o t}(t) \sum_{i=1}^{M} g_{i, j}(t) \frac{\beta_{i}^{t g t}(t)}{\sum_{k \in K_{i}} g_{i, k}(t)} \\
\Leftrightarrow \\
\hat{I}_{j}^{t o t}(t)=\frac{N_{j}(t)}{1-\sum_{i=1}^{M} \beta_{i}^{t g t}(t) \frac{g_{i, j}}{\sum_{k \in K_{i}} g_{i, k}(t)}}
\end{gathered}
$$

Solving for $\hat{\Lambda}_{j}(t)=\frac{\hat{I}_{j}^{\text {tot }}}{N_{j}}$ in the last equation above yields another practical estimate of the uplink noise rise

$$
\hat{\Lambda}_{j}^{I M R C}(t)=\frac{1}{1-\sum_{i=1}^{M} \beta_{i}^{t g t}(t) \frac{g_{i, j}}{\sum_{k \in K_{i}} g_{i, k}(t)}}
$$

where the index $(I M R C)$ indicates that we have assumed maximum ratio combining of all received signals belonging to the same user and that $I_{k}(t) \approx I_{j}(t)$. Notice the resemblance with equation (1). We thus have an explicit estimate of the uplink relative load, $L_{j}=\sum_{i=1}^{M} \beta_{i}^{t g t}(t) \frac{g_{i, j}}{\sum_{k \in K_{i}} g_{i, k}(t)}$, which is a weighted sum of the users' $\beta^{t g t}$.

Of course, we may once again choose to use selection combining between all received signals or the actual combining. This results in two more estimates

$$
\hat{\Lambda}_{j}^{I S C}(t)=\frac{1}{1-\sum_{i=1}^{M} \beta_{i}^{t g t}(t) \frac{g_{i, j}}{\max _{k \in K_{i}} g_{i, k}(t)}}
$$

and

$$
\hat{\Lambda}_{j}^{I B O T H}(t)=\frac{1}{1-\sum_{i=1}^{M} \beta_{i}^{t g t}(t) \frac{g_{i, j}}{f_{2}\left(g(t), K_{i}(t)\right)}}
$$

where $f_{2}$, much like $f$, combines the path gain values according to $K_{i}(t)$. The performance of these estimates in terms of the error's average and standard deviation is evaluated in [9].

\section{Measurements Availability}

Above, complete knowledge of the all path gain values was assumed. This, however, is not the case in practice. In this section we will define two types of limitations on the path gain knowledge. The estimates' sensitivity to these limitations will then be studied in Section IV.

\section{A. Mobile Assisted Measurements}

Users deliver path gain measurement reports to the system. These measurements represent the path loss and shadow fading the user experiences on the downlink (the fast fading is assumed to be filtered out by the users). We assume that the corresponding values on the uplink to be approximately the same with respect to path loss and shadow fading. The measurements can thus be used in the previously derived estimates. Two different ways of scheduling the measurement reports, which are both in the 3GPP standard [10], are studied herein. 
M1: The mobile stations are requested to periodically (but not necessarily synchronously) report pilot power measurements from the e.g., six strongest base stations at a rate of for example $0.5 \mathrm{~Hz}$.

M2: For handover purposes, the mobile typically reports similar measurements in an event-driven fashion. It measures the pilot powers from the neighboring cells and reports up to the six strongest path gains at handover events.

Unknown path gain values are assumed to be small and therefore set to zero.

\section{B. Distributed Information}

In reality there will be several RNC:s serving an area over which a user may move during a session. According to the 3GPP standard, a user initiating its session in one RNC, call it $R N C_{2}$, and during the session moves to an area supported by another RNC, $R N C_{1}$, will not report path gain measurements to $R N C_{1}$ but to $R N C_{2}$. A user located inside $R N C_{1}$ 's service area may thus introduce considerable interference power to the base stations without delivering any path gain reports to $R N C_{1}$. All the above estimates can only consider users reporting path gain measurements to the RNC the estimate reside in. Besides the obvious solution to neglect users not reporting their path gain, we propose a combination of the estimates assuming $I_{k}=I_{j}$ and $N_{k}=N_{j}$. Below is a derivation of the alternative estimate which estimates the noise rise in base stations belonging to $R N C_{1}$.

Split the sum over users in equation (4) into two sums, one being over the users reporting their path gain measurements to $R N C_{1}$ and the other over users reporting to other RNCs (here represented by $R N C_{2}$ ). Exemplifying with an assumption of maximum ratio combining is used for all soft handover links

$$
\begin{aligned}
I_{j}(t)=1 & +\sum_{i \in R N C_{1}} \beta_{i}^{t g t}(t) \frac{g_{i, j}(t)}{\sum_{k \in K_{i}} \frac{g_{i, k}(t)}{I_{k}(t)}} \\
& +\sum_{i \in R N C_{2}} \beta_{i}^{t g t}(t) \frac{g_{i, j}(t)}{\sum_{k \in K_{i}} \frac{g_{i, k}(t)}{I_{k}(t)}}
\end{aligned}
$$

Where cell $j$ is assumed to belong to $R N C_{1}$. Consider just the sum over users in $R N C_{2}$ and assume that $I_{k}(t)$ therein approximately equals $I_{j}(t)$. This allows us to write the sum as

$$
I_{j}(t) \sum_{i \in\left(R N C_{2}\right)} \beta_{i}^{t g t}(t) \frac{g_{i, j}(t)}{\sum_{k \in K_{i}} g_{i, k}(t)}
$$

Substituting the second sum in equation (12) with the above expression and solve for $I_{j}(t)$ yield

$$
I_{j}(t)=\frac{1+\sum_{i \in R N C_{1}} \beta_{i}^{t g t}(t) \frac{g_{i, j}(t)}{\sum_{k \in K_{i}} \frac{g_{i, k}(t)}{I_{k}(t)}}}{1-\sum_{i \in\left(R N C_{2}\right)} \beta_{i}^{t g t}(t) \frac{g_{i, j}(t)}{\sum_{k \in K_{i}} g_{i, k}(t)}}
$$

Assume that $N_{j}=N_{k}=N$ and that $\Lambda_{k}(t)=\Lambda_{k}(t-1)$. Dividing the above equation by $N$ then results in an estimate of the uplink noise rise which is a combination of equation
(6) and (9)

$$
\hat{\Lambda}_{j}^{(R N C)}(t)=\frac{1+\sum_{i \in\left(R N C_{1}\right)} \beta_{i}^{t g t}(t) \frac{g_{i, j}(t)}{\sum_{k \in K_{i}} \frac{g_{i, k}^{(t)}}{\hat{\Lambda}_{k}^{(R N C)}(t-1)}}}{1-\sum_{i \in\left(R N C_{2}\right)} \beta_{i}^{t g t}(t) \frac{g_{i, j}(t)}{\sum_{k \in K_{i}} g_{i, k(t)}}}
$$

Note that the sum in the denominator represents the load that users reporting to $R N C_{2}$ introduce to cell $j$. Neglecting these users results in a larger denominator, which yields a smaller estimate. This estimate is interesting since $R N C_{2}$ can now send a message to $R N C_{1}$ containing the additional load that users belonging to $R N C_{2}$ introduces in each cell controlled by $R N C_{1}$. This message would then be far smaller than one containing the complete path gain information regarding these users. Obviously we can choose to assume soft handover is used everywhere here as well, or we can use the actual combination of soft and softer handover. This gives us three types of estimates that uses the technique with sending relative load information between different RNCs.

\section{Simulations}

The estimates rely on measurements of the path gains between user and base station. In reality, we will not have complete knowledge of the entire path gain matrix due to a number of reasons. Therefore we have studied the estimates' performance when the path gain knowledge is limited in some sense. As a comparison, Figure 1 shows average error of the three estimates when knowing the whole uplink path gain matrix and actual received $\beta_{i}$. The error is caused by a number of factors [9].

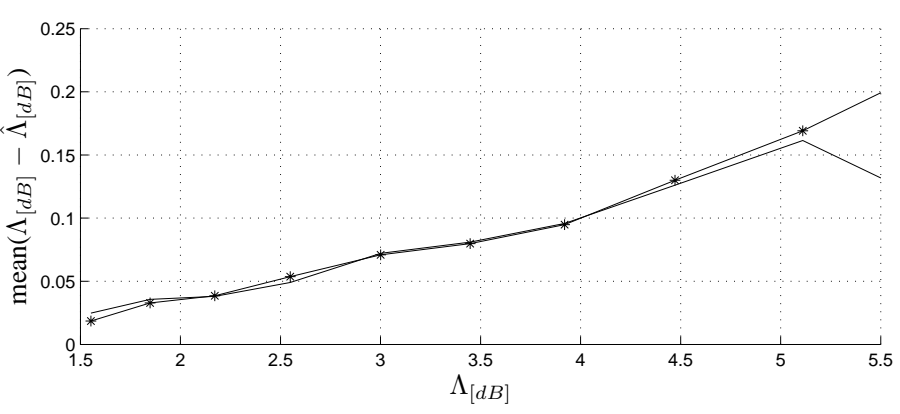
Fig. 1. Mean error when knowing the entire path gain matrix.
${ }^{*}: \Lambda^{(N B O T H)},{ }^{(N}, \Lambda^{(I B O T H)}$

\section{A. Measurement Report Frequency}

Consider a simulation area consisting of 21 cells, all serviced by the same RNC. Not surprisingly, as can be seen in Figure 2, using event driven pathgain reports (i.e., M2 above) results in far less reports per user and hence less signaling overhead for the system. More interesting is the fact that using M2 does not necessarily imply a worse estimate (in fact, the periodicity of M1 was selected to obtain comparable performance), see Figure 3, 4 and 5 which show the average estimation error of $\hat{\Lambda}^{(N M R C)}, \hat{\Lambda}^{(N S C)}$ and $\hat{\Lambda}^{(N B O T H)}$ when using users travelling at an average speed of 10, 20 and $70 \mathrm{~km} / \mathrm{h}$, respectively. A comparison between the estimates assuming $I_{k}=I_{j}$ instead of $N_{k}=N_{j}$, shows that there is 
no statistical difference between the two in the average error. The standard deviation, however, is considerably larger when using $\Lambda^{I M R C}, \Lambda^{I S C}$ or $\Lambda^{I B O T H}$ in conjunction with M1, see Figure 6 and 7.

Only when travelling at a rather high speed, $70 \mathrm{~km} / \mathrm{h}$, using M1 or M2 results in different estimation error statistics. At this speed, M1 provides a better average error but as can be seen in Figure 6, the standard deviation is much higher compared with using M2. The average error may be canceled by an error correction method, but can do almost nothing to combat a high standard deviation. Average error cancelation also requires a low standard deviation, which is why M2 can be considered providing an even better estimate than M1.

It is the users that are close to the cell boarder that are the most important ones to have somewhat accurate path gain knowledge of since these are the users that cause most of the inter-cell-interference. Since these users are more likely to change their soft handover setup compared to users within the cell, it is also more likely that we get a report from these users when using $\mathrm{M} 2$.

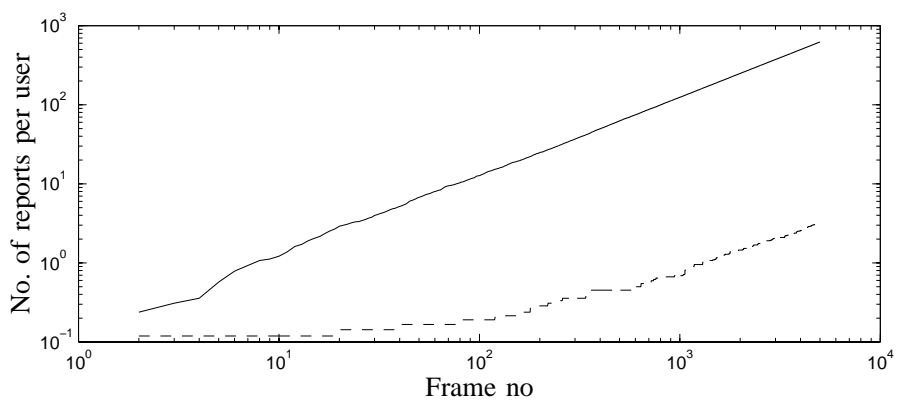

Fig. 2. Cumulative sum over number of reports per user for M1 (solid) and M2 (dashed)

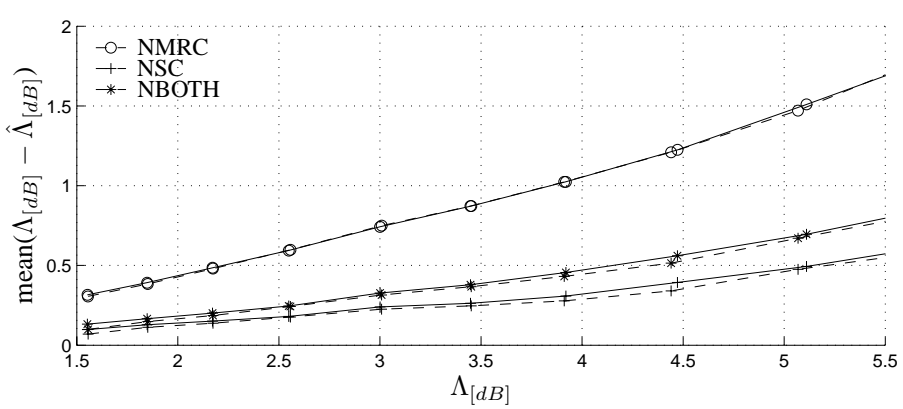

Fig. 3. Mean error. User speed: $10 \mathrm{~km} / \mathrm{h}$. Solid:M1, dashed:M2.

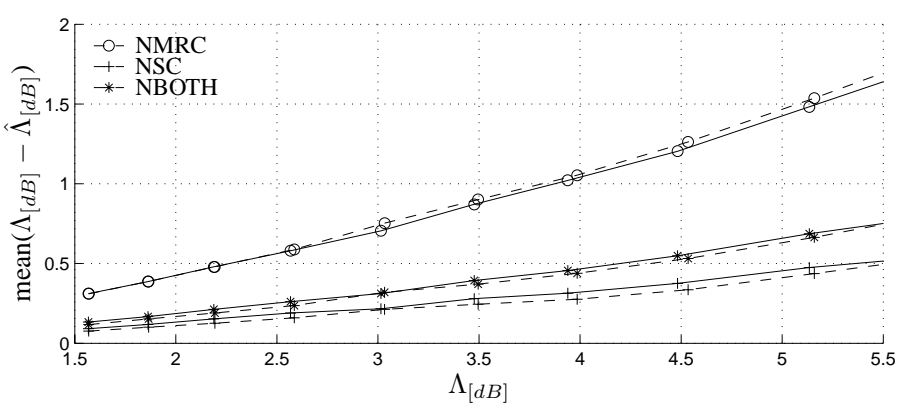

Fig. 4. Mean error. User speed: $20 \mathrm{~km} / \mathrm{h}$. Solid:M1, dashed:M2.

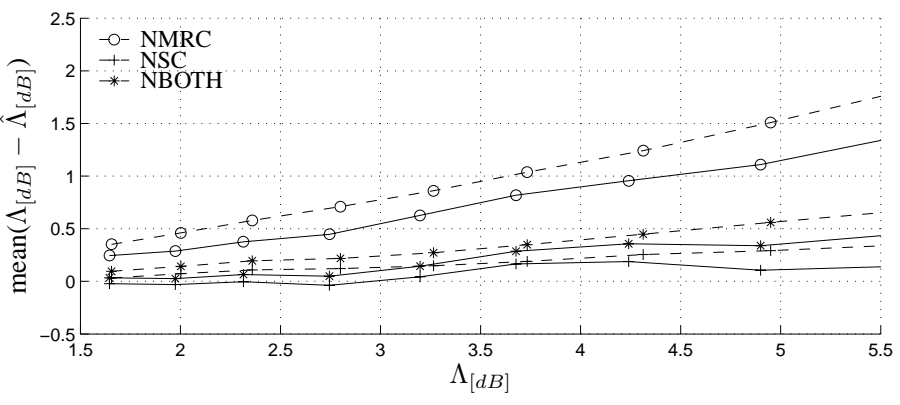

Fig. 5. Mean errors. User speed: 70 km/h. Solid:M1, dashed:M2.

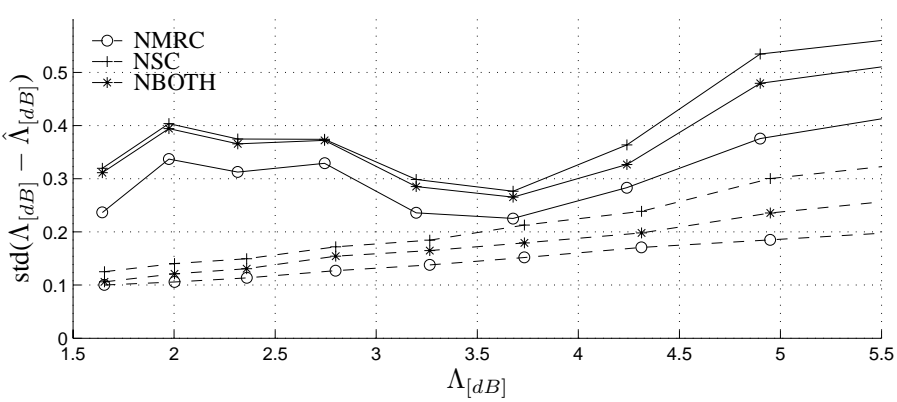

Fig. 6. Standard deviation of single errors. All is for $70 \mathrm{~km} / \mathrm{h}$. solid:M1, dashed:M2

\section{B. Several Radio Network Controllers}

Another, even more drastic way, of constraining the available path gain knowledge in the RNC is to provide reports from only a subset of the users. As argued in Section III, some users will not report their path gain, even though they are power controlled by this RNC, since they started their session when located in an area served by another RNC. In this subsection we have used path gain reports according to M2 above and compared the performance of the estimate defined by equation (13) for different fractions of external users (i.e., users not reporting their path gain to the RNC referred to as $R N C_{1}$ above). As a comparison we have also computed statistics for the estimates that completely ignores the external users. Figure 8, 9 and 10 show the average estimation error when 10,20 and $30 \%$ of the users report to another RNC than the one they are closest to, respectively. Clearly, just ignoring that some users are not considered in the estimate can be quite drastic. However, when using the estimate that incorparates an estimate of the load contribution from the external users, the performance is almost equal to the case where measurement

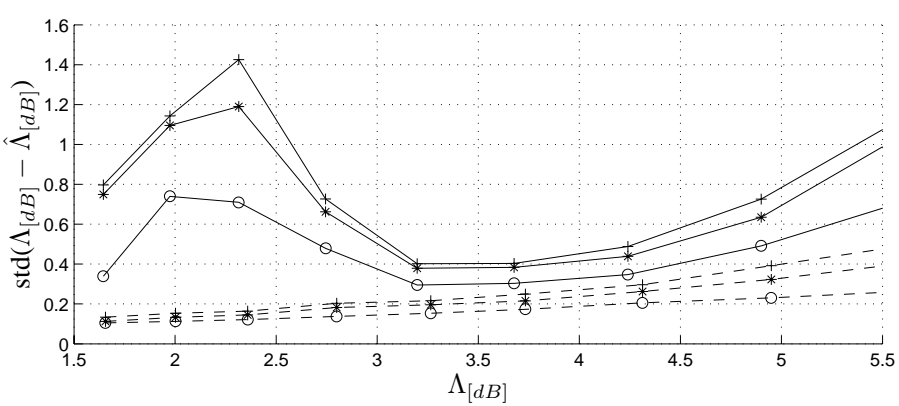

Fig. 7. Standard deviation of single errors. 'o': $\Lambda^{I M R C}$, '+': $\Lambda^{I S C}$, '*': $\Lambda^{I \text { IOT H}}$. All is for $70 \mathrm{~km} / \mathrm{h}$. solid:M1, dashed:M2 
reports from all users are available. Figure 11 shows that these estimates have approximately the same standard deviation as the ones assuming equal background noise power in all cells. The estimates assuming $I_{k}=I_{j}$ are not shown here. The problem of not receiving path gain reports from some users can be solved in the same way, i.e., with small messages between different RNCs, without any additional changes to the estimtes.

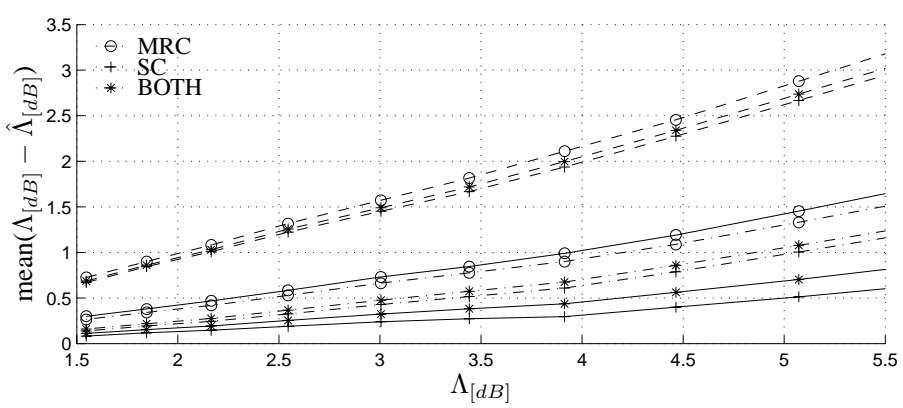

Fig. 8. Mean errors. Solid:All reports, dashed:Reports in one RNC, dashdotted: $\hat{\Lambda}^{(R N C)} .10 \%$ of the users report to another RNC.

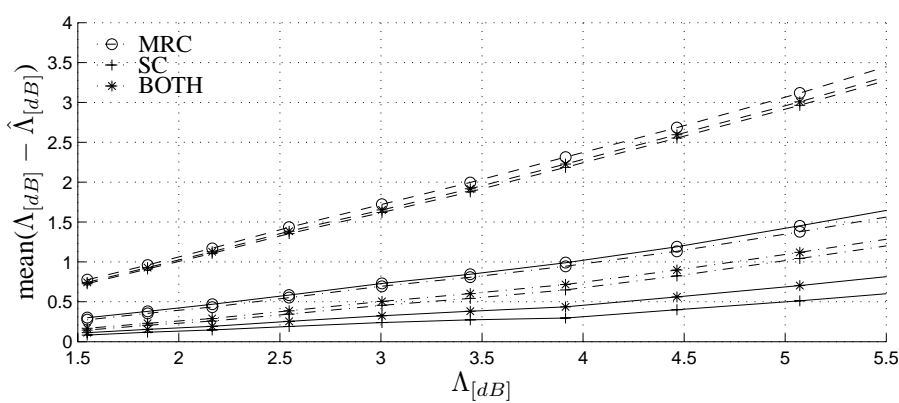

Fig. 9. Mean errors. Solid:All reports, dashed:Reports in one RNC, dashdotted: $\hat{\Lambda}^{(R N C)} \cdot 20 \%$ of the users report to another RNC.

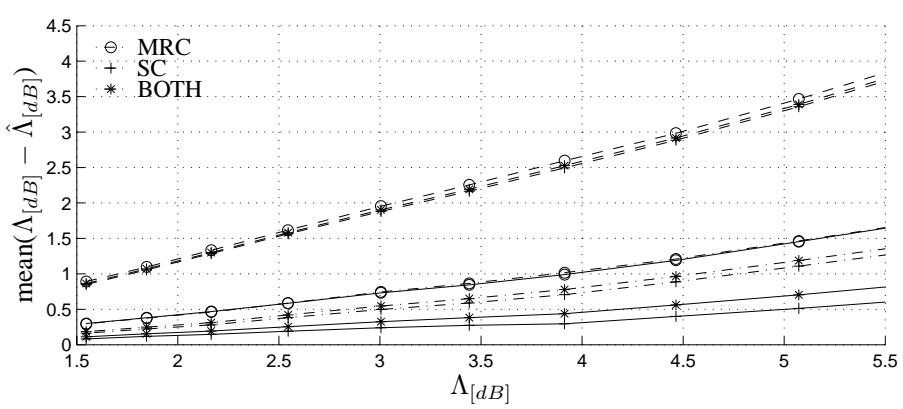

Fig. 10. Mean errors. Solid:All reports, dashed:Reports in one RNC, dashdotted: $\hat{\Lambda}^{(R N C)} .30 \%$ of the users report to another RNC.

\section{CONCLUSIONS}

We have proposed and studied a number of uplink noise rise estimates. The estimates use path gain measurements which are readily available to the system. Two different scheduling approaches of path gain measurement reports were studied. Using event-triggered reports requires less signaling overhead while providing noise rise estimates which have equal or less variance compared to using periodically delivered reports. Only the part of the study where all users were travelling at a high speed $(70 \mathrm{~km} / \mathrm{h})$ the average estimation error was larger

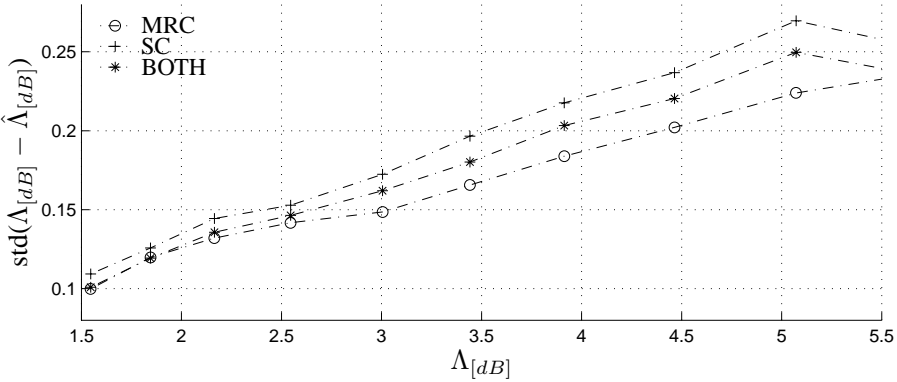

Fig. 11. Standard deviation of single errors. Dashdotted: $\hat{\Lambda}^{(R N C)} \cdot 30 \%$ of the users report to another RNC.

when using event-triggered reports compared with periodically delivered reports. However, since the standard deviation of the estimates' error is lower when using event-triggered reports and a low standard deviation is a requirement for error compensation, using event-triggered reports is recommended according to this study.

The estimates' sensitivity to incomplete path gain knowledge due to missing path gain reports was also investigated through simulations. When using an estimate that incorparates information about the additional relative load in one RNC which is caused by users belonging to in another RNC, it is possible to estimate the uplink noise rise with acceptable performance even with considerably limited path gain knowledge.

\section{REFERENCES}

[1] C. Y. Huang and R. D. Yates, "Call admission in power controlled CDMA systems," in Proc. IEEE Vehicular Technology Conference, Atlanta, GA, USA, May 1996.

[2] F. Gunnarsson, E. Geijer Lundin, G. Bark, N. Wiberg, "Uplink admission control in WCDMA based on relative load estimates," in Proc. IEEE International Conference on Communications, New York, USA, April 2002.

[3] H. Holma and J. Laakso, "Uplink admission control and soft capacity with MUD in CDMA," in Proc. IEEE Vehicular Technology Conference, Amsterdam, the Netherlands, Sep 1999.

[4] A. Abrardo, G. Benelli, G. Giambene, and D. Sennati, "Performance analysis of a packet scheduling policy for a DSCDMA cellular system," in Proc. IEEE Vehicular Technology Conference, Rhodes, Greece, May 2001.

[5] W. Ying, Z. Jingmei, W. Weidong, and Z. Ping, "Call admission control in hierarchi cell structure," in Proc. IEEE Vehicular Technology Conference, Birmingham, AL, USA, may 2002.

[6] A. Viterbi and A. Viterbi, "Erlang capacity of a power controlled CDMA system," IEEE Journal on Selected Areas in Communications, pp. 892-900, 1993.

[7] J. Sanchez, J. Perez-Romero, O. Sallent, and R. Agusti, "Mixing conversational and interactive traffic in the UMTS radio access network," in Proc.IEEE 4th International Workshop on Mobile and Wireless Communications Network, 2002, Sep 2002, pp. 597-601.

[8] K. Hiltunen, N. Binucci, and J. Bergstrom, "Comparison between the periodic and event-triggered intra-frequency handover measurement reporting in WCDMA," in Proc. IEEE wireless Communications and Networking Conference, Chicago, IL, USA, Sep 2000.

[9] E. Geijer Lundin, F. Gunnarsson, F. Gustafsson, "Uplink load estimation in WCDMA," in Proc. IEEE Wireless Communications and Networking Conference, New Orleans, USA, March 2003.

[10] 3GPP, "RRC Protocol Specification," Technical Specification 3GPP TS 25.331, 1999. 